\title{
APPLICATION OF SOFT P-OPEN SET TO BINARY SOFT STRUCTURES
}

\author{
Arif Mehmood Khattak ${ }^{1}$, Zia Ul-Haq ${ }^{2}$, Zamir Barki ${ }^{3}$, Muhammad Ilyas ${ }^{4}$ \\ ${ }^{1}$ Department of Mathematics and Statistics Riphah International University, Sector I-14, Islamabad \\ 'Department of Mathematics, Bannu University of Science and Technology, Bannu, Pakistan. \\ *Corresponding Author Email: mehdaniyal@gmail.com
}

This is an open access article distributed under the Creative Commons Attribution License, which permits unrestricted use, distribution, and reproduction in any medium, provided the original work is properly cited.

\section{ARTICLE DETAILS}

\section{Article History:}

Received 26 June 2018 Accepted 2 July 2018 Available online 1 August 2018

\section{ABSTRACT}

The main aim of this paper is to introduce a single structure which carries the subsets of $X$ as well as the subsets of $\mathrm{Y}$ under the parameter E for studying the information about the ordered pair of soft subsets of X and Y. Such a structure is called a binary soft structure from $\mathrm{X}$ to $\mathrm{Y}$. The purpose of this paper is to introduce certain binary soft weak axioms that are analogous to the axioms of topology.

\section{KEYWORDS}

Binary soft topology, binary soft weak open sets. binary soft weak closed sets, binary soft weak separation axioms and binary soft $\mathrm{T}_{0}$ space with respect to coordinates.

\section{INTRODUCTION}

The concept of soft sets was first introduced by Molodtsov in 1999 as a general mathematical technique for dealing with uncertain substances [1]. In, Molodtsov magnificently applied the soft theory in numerous ways, such as smoothness of functions, game theory, operations research, Riemann integration, Perron integration, probability, theory of measurement, and so on [1,2]. Point soft set topology deals with a nonempty setX to gether with a collection $\tau$ of sub set X under some set of parameters satisfying certain conditions. Such a collection $\tau$ is called a soft topological structure onX.

In 2016 Ahu Acikgöz and Nihal Tas introduced the notion of binary soft set Theory on two master sets and studied some basic characteristics [3]. In prolongation, a group researcher planned the idea of binary soft topology and linked fundamental properties which are defined over two master sets with appropriate parameters [4]. Other researchers threw their detailed discussion on Binary Soft Topological [5]. Dr. A. Kalaichelvi and P.H. Malini beautifully discussed Application of Fuzzy Soft Sets to Investment Decision and also discussed some more results related to this particular field [6]. N. Y. Özgür and N. Taş, studied some more Application of Fuzzy Soft Sets to Investment Decision Making Problem [7]. N. Taş, N. Y. Özgür and P. Demir worked over An Application of Soft Set and Fuzzy Soft Set Theories to Stock Management J. C. R. Alcantud et al carefully discussed Valuation Fuzzy Soft Sets: A Flexible Fuzzy Soft Set Based Decision-Making Procedure for the Valuation of Assets N. Çağman, S [8-10].

Enginoğlu attractively explored Soft Matrix Theory and some very basic results related to it and Its Decision Making In continuation, in the present paper binary soft topological structures known as soft weak structures with respect to first coordinate as well as with respect to second coordinate are defined. Moreover, some basic results related to these structures are also planted in this paper. The same structures are defined over soft points of binary soft topological structure and related results are also reflected here with respect to ordinary and soft points.

\section{PRELIMINARIES}

Definition 1: [11]. Let $X$ be an initial universe and let $E$ be a set of parameters. Let $\mathrm{P}(\mathrm{X})$ denote the power set of $\mathrm{X}$ and let $\mathrm{A}$ be a non-empty subset ofE. A pair $(F, A)$ iscalled a soft set overX, where $F$ is a mapping given by: $A \rightarrow P(X)$. In other words, a soft set over $X$ is a parameterized family of subsets of the universe X. For $\varepsilon \in A, F(\varepsilon)$ may be considered as the set of $\varepsilon$-approximate elements of the soft set (F, A). Clearly, a soft set is not a set.

Let $U_{1}, U_{2}$ be two initial universe sets and $E$ be a set of parameters.

LetP $\left(U_{1}\right), P\left(U_{2}\right)$ denote the power set of $U_{1}, U_{2}$ respectively. Also, letA, $\mathrm{B}, \mathrm{C} \subseteq \mathrm{E}$.

Definition 2: [3]. A pair (F, A) is said to be a binary soft set over $\mathrm{U}_{1}, \mathrm{U}_{2}$ where $\mathrm{F}$ is defined as below:

$\mathrm{F}: \mathrm{A} \rightarrow \mathrm{P}\left(\mathrm{U}_{1}\right) \times \mathrm{P}\left(\mathrm{U}_{2}\right), \mathrm{F}(\mathrm{e})=(\mathrm{X}, \mathrm{Y})$ for each e $\in \mathrm{A}$ such that $\mathrm{X} \subseteq \mathrm{U}_{1}, \mathrm{Y} \subseteq$ $\mathrm{U}_{2}$

Definition 3: [3]. A binary soft set (F, A) over $U_{1}, U_{2}$ is called a binary absolute soft set, denoted by $\widetilde{\widetilde{A}}$ if $\mathrm{F}(\mathrm{e})=\left(\mathrm{U}_{1}, \mathrm{U}_{2}\right)$ for eache $\in$ A.

Definition 4: [3]. The intersection of two binary soft sets of (F, A) and (G, B) over the common $U_{1}, U_{2}$ is the binary soft set $(H, C)$, where $C=A \cap B$ and for all $\mathrm{e} \in \mathrm{C}$

$$
H(e)=\left\{\begin{array}{c}
\left(X_{1}, Y_{1}\right) \text { if } e \in A-B \\
\left(X_{2}, Y_{2}\right) \text { if } e \in B-A \\
\left(X_{1} \cup X_{2}, Y_{1} \cup Y_{2}\right) \text { if } e \in A \cap B
\end{array}\right.
$$

Such that $\mathrm{F}(\mathrm{e})=\left(\mathrm{X}_{1}, \mathrm{Y}_{1}\right)$ for each $\mathrm{e} \in \mathrm{A}$ and $\mathrm{G}(\mathrm{e})=\left(\mathrm{X}_{2}, \mathrm{Y}_{2}\right)$ for eache $\in \mathrm{B}$. We denote it $(F, A) \widetilde{U}(G, A)=(H, C)$

Definition 5: [3]. The intersection of two binary soft sets (F, A) and (G, B) over a common $U_{1}, U_{2}$ is the binary soft set $(H, C)$, whereC $=A \cap B$, and $\left.\mathrm{H}(\mathrm{e})=\left(\mathrm{X}_{1} \cap \mathrm{X}_{2}, \mathrm{Y}_{1} \cap \mathrm{Y}_{2}\right)\right)$ for each e $\in \mathrm{C}$ such that $\mathrm{F}(\mathrm{e})=\left(\mathrm{X}_{1}, \mathrm{Y}_{1}\right)$ for each $\mathrm{e} \in \mathrm{A}$ and $\mathrm{G}(\mathrm{e})=\left(\mathrm{X}_{2}, \mathrm{Y}_{2}\right)$ for eache $\in \mathrm{B}$. We denote it as $(\mathrm{F}, \mathrm{A}) \widetilde{\tilde{n}}(\mathrm{G}, \mathrm{B})=(\mathrm{H}, \mathrm{C})$

Definition 6: [3]. Let (F, A) and (G, B) be two binary soft sets over a common $U_{1}, U_{2} .(F, A)$ is called a binary soft subset of $(G, B)$ if (i) $\mathrm{A} \subseteq \mathrm{B}$,

(ii) $X_{1} \subseteq X_{2}$ and $Y_{1} \subseteq Y_{2}$ Such that $F(e)=\left(X_{1}, Y_{1}\right), G(e)=\left(X_{2}, Y_{2}\right)$ for eache $\in$ A. We denote it as $(F, A) \widetilde{\widetilde{\subseteq}}(G, B)$.

Definition 7: [3]. A binary soft set $(F, A)$ over $U_{1}, U_{2}$ is called a binary null soft set, denoted by if $\mathrm{F}(\mathrm{e})=(\varphi, \varphi)$ for eache $\in \mathrm{A}$.

Definition 8: [3]. The difference of two binary soft sets (F, A) and (G, A) 
over the Common $U_{1}, U_{2}$ is the binary soft set $(H, A)$, where $H(e)\left(X_{1}-\right.$ $\left.\mathrm{X}_{2}, \mathrm{Y}_{1}-\mathrm{Y}_{2}\right)$ for each $\mathrm{e} \in \mathrm{A}$ such that $(\mathrm{F}, \mathrm{A})=\left(\mathrm{X}_{1}, \mathrm{Y}_{1}\right)$ and $(\mathrm{G}, \mathrm{A})=\left(\mathrm{X}_{2}, \mathrm{Y}_{2}\right)$.

Definition 9: [4]. Let $\tau_{\Delta}$ be the collection of binary soft sets over $U_{1}, U_{2}$ then $\tau_{\Delta}$ is said to be a binary soft topology on $U_{1}, U_{2}$ if

(i) $\widetilde{\widetilde{\varphi}}, \widetilde{\mathrm{X}} \in \tau_{\Delta}$

(ii) The union of any member of binary soft sets in $\tau_{\Delta}$ belongs to $\tau_{\Delta}$.

(iii) The intersection of any two binary soft sets in $\tau_{\Delta}$ belongs to $\tau_{\Delta}$.

Then $\left(U_{1}, U_{2}, \tau_{\Delta}, E\right)$ is called a binary soft topological space over $U_{1}, U_{2}$.

Definition 10: Let $(F, A)$ be any binary soft sub set of a binary soft topological space $(\mathrm{X}, \mathrm{Y}, \tau, \mathrm{E})$ then $(\mathrm{F}, \mathrm{A})$ is called

1) Binary soft pre-open set of $(X, Y, \tau, E)$ if $(F, A) \subseteq$ in $(\mathrm{cl}((F, A)))$ and 2) Binary soft pre-closed set of $(X, Y, \tau, E)$ if $(F, A) \supseteq \operatorname{cl}(\operatorname{int}(F, A)))$.

The set of all binary P-open soft sets is denoted by BSPO (U) and the set of all binary P-closed sets is denoted by BSCO (U).

\section{BINARY SOFT WEAK SEPARATION AXIOMS}

In this section binary soft weak separation axioms in Binary Soft Topological Spaces are reflected.

Definition 11: A binary soft topological space $(\widetilde{\mathrm{X}}, \widetilde{\mathrm{Y}}, \mathcal{M}, \mathrm{A})$ is called a binary soft $\mathrm{P}_{0}$ space if for any two binary soft points $\left(\mathrm{x}_{1}, \mathrm{y}_{1}\right),\left(\mathrm{x}_{2}, \mathrm{y}_{2}\right) \tilde{\varepsilon}(\widetilde{\mathrm{X}}, \widetilde{\mathrm{Y}})$ such that $\mathrm{x}_{1}>\mathrm{x}_{2}, \mathrm{y}_{1}>\mathrm{y}_{2}$ there exists binary soft $\mathrm{p}$-open sets $\left(\mathrm{F}_{1}, \mathrm{~A}\right)$ and

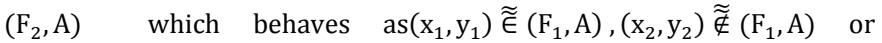
$\left(\mathrm{x}_{2}, \mathrm{y}_{2}\right) \widetilde{\in}\left(\mathrm{F}_{2}, \mathrm{~A}\right)$ and $\left(\mathrm{x}_{1}, \mathrm{y}_{1}\right) \widetilde{\notin}\left(\mathrm{F}_{2}, \mathrm{~A}\right)$.

Definition 12: A binary soft topological space $(\widetilde{\mathrm{X}}, \widetilde{\mathrm{Y}}, \mathcal{M}, \mathrm{A})$ is called a binary soft $\mathrm{P}_{1}$ space if for any two binary soft points $\left(\mathrm{x}_{1}, \mathrm{y}_{1}\right),\left(\mathrm{x}_{2}, \mathrm{y}_{2}\right) \tilde{\mathcal{E}}(\widetilde{\mathrm{X}}, \widetilde{\mathrm{Y}})$ such that $x_{1}>x_{2}, y_{1}>y_{2}$ If there exists binary soft p-open sets $\left(F_{1}, A\right)$ and $\left(\mathrm{F}_{2}, \mathrm{~A}\right)$ which behaves as $\left(\mathrm{x}_{1}, \mathrm{y}_{1}\right) \widetilde{\in}\left(\mathrm{F}_{1}, \mathrm{~A}\right)$ and $\left(\mathrm{x}_{2}, \mathrm{y}_{2}\right) \widetilde{\notin}\left(\mathrm{F}_{1}, \mathrm{~A}\right)$ and $\left(\mathrm{x}_{2}, \mathrm{y}_{2}\right) \widetilde{\in}\left(\mathrm{F}_{2}, \mathrm{~A}\right)$ and $\left(\mathrm{x}_{1}, \mathrm{y}_{1}\right) \widetilde{\notin}\left(\mathrm{F}_{2}, \mathrm{~A}\right)$.

Definition 13: Two binary soft p-open sets ( $(F, A),(G, A))$ and (H, A), (I, A) are said to be disjoint if $((\mathrm{F}, \mathrm{A}) \sqcap(\mathrm{H}, \mathrm{A}),(\mathrm{G}, \mathrm{A}) \sqcap(\mathrm{I}, \mathrm{A}))=(\Phi, \Phi)$. That is $(\mathrm{F}, \mathrm{A}) \sqcap(\mathrm{H}, \mathrm{A})=(\Phi, \Phi)$ and $(\mathrm{G}, \mathrm{A}) \sqcap(\mathrm{I}, \mathrm{A})=(\Phi, \Phi)$.

Definition 14: A binary soft topological space $(\widetilde{X}, \widetilde{Y}, \mathcal{M}, A)$ is called a binary soft $\mathrm{P}_{2}$ space if for any two binary soft points $\left(\mathrm{x}_{1}, \mathrm{y}_{1}\right),\left(\mathrm{x}_{2}, \mathrm{y}_{2}\right) \tilde{\mathcal{E}}(\widetilde{\mathrm{X}}, \widetilde{\mathrm{Y}})$ such that $\mathrm{x}_{1}>\mathrm{x}_{2}, \mathrm{y}_{1}>\mathrm{y}_{2}$ If there exists binary soft p-open sets $\left(\mathrm{F}_{1}, \mathrm{~A}\right)$ and $\left(\mathrm{F}_{2}, \mathrm{~A}\right)$ which behaves as $\left(\mathrm{x}_{1}, \mathrm{y}_{1}\right) \widetilde{\widetilde{\epsilon}}\left(\mathrm{F}_{1}, \mathrm{~A}\right)$ and $\left(\mathrm{x}_{2}, \mathrm{y}_{2}\right) \widetilde{\widetilde{\epsilon}}\left(\mathrm{F}_{2}, \mathrm{~A}\right)$ and moreover $\left(F_{1}, A\right)$ and $\left(F_{2}, A\right)$ are disjoint.

Definition 15: A binary soft topological space $(\widetilde{X}, \widetilde{Y}, \tau \times \sigma, A)$ is called a binary soft $\mathrm{P}_{0}$ with respect to the first coordinate if for every pair of binary points $\left(\mathrm{x}_{1}, \alpha\right),\left(\mathrm{y}_{1}, \alpha\right)$ there exists $((\mathrm{F}, \mathrm{A}),(\mathrm{G}, \mathrm{A})) \tilde{\varepsilon} \tau \times \sigma$ withx $_{1} \tilde{\varepsilon}(F, A), y_{1} \widetilde{\notin}(F, A), \alpha \tilde{\varepsilon}(G, A)$.where p-open $(F, A)$ 'in $\tau$ and p- open $(\mathrm{G}, \mathrm{A})$ in $\sigma$.

Definition 16: A binary soft topological space $(\widetilde{X}, \widetilde{Y}, \tau \times \sigma, A)$ is called a binary soft $P_{0}$ with respect to the second coordinate if for every pair of binary points $\left(\beta, \mathrm{x}_{2}\right),\left(\beta, \mathrm{y}_{2}\right)$ there exists $((\mathrm{F}, \mathrm{A}),(\mathrm{G}, \mathrm{A})) \tilde{\varepsilon} \tau \times \sigma$ $\operatorname{with} \beta \tilde{\varepsilon}(\mathrm{F}, \mathrm{A}), \mathrm{x}_{2} \tilde{\mathcal{E}}(\mathrm{G}, \mathrm{A}), \mathrm{y}_{2} \widetilde{\notin}(\mathrm{G}, \mathrm{A})$. where p-open $(\mathrm{F}, \mathrm{A})$ 'in $\tau$ and p- 'open $(\mathrm{G}, \mathrm{A})$ in $\sigma . e_{\mathbb{G}}, e_{\mathbb{H}}$

Definition 17: A binary soft topological space $(\widetilde{\mathrm{X}}, \widetilde{\mathrm{Y}}, \mathcal{M}, \mathrm{A})$ is called a binary soft $\mathrm{P}_{0}$ space if for any two binary soft points $\left(e_{\mathbb{G}_{1}}, e_{\mathbb{H}_{1}}\right),\left(e_{\mathbb{G}_{2}}, e_{\mathbb{H}_{2}}\right) \tilde{\varepsilon}\left(\widetilde{\mathrm{X}_{\mathrm{A}}}, \widetilde{\mathrm{Y}_{\mathrm{A}}}\right)$ such that $e_{\mathbb{G}_{1}}>e_{\mathbb{G}_{2}}, e_{\mathbb{H}_{1}}>e_{\mathbb{H}_{2}}$ there exists binary soft p-open sets $\left(\mathrm{F}_{1}, \mathrm{~A}\right)$ and $\left(\mathrm{F}_{2}, \mathrm{~A}\right)$ which behaves $\operatorname{as}\left(e_{\mathbb{G}_{1}}, e_{\mathbb{H}_{1}}\right) \widetilde{\epsilon}\left(\mathrm{F}_{1}, \mathrm{~A}\right),\left(e_{\mathbb{G}_{2}}, e_{\mathbb{H}_{2}}\right) \widetilde{\widetilde{\epsilon}}\left(\mathrm{F}_{1}, \mathrm{~A}\right)$ or $\left(e_{\mathbb{G}_{2}}, e_{\mathbb{H}_{2}}\right) \widetilde{\in}\left(\mathrm{F}_{2}, \mathrm{~A}\right)$ and $\left(e_{\mathbb{G}_{1}}, e_{\mathbb{H}_{1}}\right) \widetilde{\widetilde{\notin}}\left(\mathrm{F}_{2}, \mathrm{~A}\right)$.

Definition 18: A binary soft topological space $(\widetilde{\mathrm{X}}, \widetilde{\mathrm{Y}}, \mathcal{M}, A)$ is called a binary soft $P_{1}$ space if for any two binary soft points $\left(e_{\mathbb{G}_{1}}, e_{\mathbb{H}_{1}}\right),\left(e_{\mathbb{G}_{2}}, e_{\mathbb{H}_{2}}\right) \tilde{\varepsilon}\left(\widetilde{X_{A}}, \widetilde{Y_{A}}\right)$ such that $e_{\mathbb{G}_{1}}>e_{\mathbb{G}_{2}}, e_{\mathbb{H}_{1}}>e_{\mathbb{H}_{2}}$ If there exists binary soft p-open sets $\left(F_{1}, A\right)$ and $\left(F_{2}, A\right)$ which behaves $\operatorname{as}\left(e_{\mathbb{G}_{1}}, e_{\mathbb{H}_{1}}\right) \widetilde{\widetilde{\epsilon}}\left(F_{1}, A\right)$ and $\left(e_{\mathbb{G}_{2}}, e_{\mathbb{H}_{2}}\right) \widetilde{\widetilde{\epsilon}}\left(F_{1}, A\right)$ and $\left(e_{\mathbb{G}_{2}}, e_{\mathbb{H}_{2}}\right) \widetilde{\widetilde{\epsilon}}\left(F_{2}, A\right)$ and $\left(e_{\mathbb{G}_{1}}, e_{\mathbb{H}_{1}}\right) \widetilde{\Psi}\left(F_{2}, A\right)$.

Definition 19: A binary soft topological space $(\tilde{X}, \tilde{Y}, \mathcal{M}, A)$ is called a binary soft $P_{2}$ space if for any two binary soft points $\left(e_{\mathbb{G}_{1}}, e_{\mathbb{H}_{1}}\right),\left(e_{\mathbb{G}_{2}}, e_{\mathbb{H}_{2}}\right) \tilde{\varepsilon}\left(\widetilde{X_{A}}, \widetilde{Y_{A}}\right)$ such that $e_{\mathbb{G}_{1}}>e_{\mathbb{G}_{2}}, e_{\mathbb{H}_{1}}>e_{\mathbb{H}_{2}}$ If there exists binary soft p-open sets $\left(F_{1}, A\right)$ and $\left(F_{2}, A\right)$ which behaves $\operatorname{as}\left(e_{\mathbb{G}_{1}}, e_{\mathbb{H}_{1}}\right) \widetilde{\in}\left(F_{1}, A\right) \quad$ and $\quad\left(e_{\mathbb{G}_{2}}, e_{\mathbb{H}_{2}}\right) \widetilde{\in}\left(F_{2}, A\right)$ and moreover
$\left(F_{1}, A\right)$ and $\left(F_{2}, A\right)$ are disjoint.

Definition 20: A binary soft topological space $(\tilde{X}, \tilde{Y}, \tau \times \sigma, A)$ is called a binary soft $P_{0}$ with respect to the first coordinate if for every pair of binary points $\left(e_{\mathbb{G}_{1}}, \alpha\right),\left(e_{\mathbb{H}_{1}}, \alpha\right)$ there exists $((F, A),(G, A)) \tilde{\mathcal{E}} \tau \times \sigma$ with $e_{\mathbb{G}_{1}} \tilde{\varepsilon}(F, A), e_{\mathbb{H}_{1}} \widetilde{\notin}(F, A), \alpha \tilde{\varepsilon}(G, A)$.where p-open $(F, A)$ 'in $\tau$ and popen $(G, A)$ in $\sigma$.

Definition 21: A binary soft topological space $(\tilde{X}, \tilde{Y}, \tau \times \sigma, A)$ is called a binary soft $P_{0}$ with respect to the second coordinate if for every pair of binary points $\left(\beta, e_{\mathbb{G}_{2}}\right),\left(\beta, e_{\mathbb{H}_{2}}\right)$ there exists $((F, A),(G, A)) \tilde{\mathcal{E}} \tau \times \sigma$ with $\beta \tilde{\varepsilon}(F, A), e_{\mathbb{G}_{2}} \tilde{\varepsilon}(G, A), e_{\mathbb{H}_{2}} \widetilde{\notin}(G, A)$. where p-open $(F, A)^{`}$ in $\tau$ and popen $(G, A)$ in $\sigma$.

\section{BINARY SOFT STRUCTURES WITH RESPECT TO ORDINARY POINTS}

Theorem 1. If the binary soft topological space $(\tilde{X}, \tilde{Y}, \rho \times \sigma, A)$ is a binary $\operatorname{soft} P_{0}$, then $(\tilde{X}, \rho, A)$ and $(\tilde{Y}, \sigma, A)$ are $\operatorname{soft} P_{0}$.

Proof. We suppose $(\tilde{X}, \tilde{Y}, \rho \times \sigma, A)$ is a binary soft $P_{0}$. Suppose $x_{1}, x_{2} \tilde{\varepsilon} \tilde{X}$ and $y_{1}, y_{2} \tilde{\varepsilon} \tilde{Y}$ with such that $x_{1}>x_{2}, y_{1}>y_{2}$.Since $(\tilde{X}, \tilde{Y}, \rho \times \sigma, A)$ is a binary soft $P_{0}$,accordingly there binary soft p-open set $((F, A),(G, A))$ such that $\quad\left(x_{1}, y_{1}\right) \tilde{\mathcal{E}}((F, A),(G, A)) ;\left(x_{2}, y_{2}\right) \tilde{\mathcal{E}}\left(F^{C}, A\right),\left(G^{C}, A\right) \quad$ or $\left(x_{1}, y_{1}\right) \tilde{\varepsilon}\left(\left(F^{C}, A\right),\left(G^{C}, A\right)\right) ;\left(x_{2}, y_{2}\right) \tilde{\varepsilon}((F, A),(G, A))$.This implies that either $x_{1} \tilde{\varepsilon}(F, A) ; x_{2} \tilde{\varepsilon}\left(F^{C}, A\right) ; y_{1} \tilde{\varepsilon}(G, A) ; y_{2} \tilde{\varepsilon}\left(G^{C}, A\right)$;or

$x_{1} \tilde{\varepsilon}\left(F^{C}, A\right) ; y_{1} \tilde{\varepsilon}\left(G^{C}, A\right) ; y_{2} \tilde{\varepsilon}(G, A)$. This implies either $x_{1} \tilde{\varepsilon}(F, A) ; x_{2} \tilde{\varepsilon}\left(F^{C}, A\right)$ or $\quad x_{1} \tilde{\varepsilon}\left(F^{C}, A\right) ; x_{1} \tilde{\varepsilon}(F, A)$ and $\quad$ either $y_{1} \tilde{\varepsilon}(G, A) ; y_{2} \tilde{\varepsilon}\left(G^{C}, A\right)$ or $_{1} \tilde{\varepsilon}\left(G^{C}, A\right) ; y_{2} \tilde{\varepsilon}(G, A)$. Since $((F, A),(G, A)) \tilde{\varepsilon} \rho \times$ $\sigma$, We have p- open $(F, A) \tilde{\varepsilon} \rho$ and p-open $(F, A) \widetilde{\mathcal{E}} \sigma$. this proves that $(\tilde{X}, \rho, A)$ and $(\tilde{Y}, \sigma, A)$ are soft $P_{0}$.

Theorem 2. A binary soft topological space $(\tilde{X}, \tilde{Y}, \tau \times \sigma, A)$ is binary soft $P_{0}$ space with respect to first and the second coordinates, then $(\tilde{X}, \tilde{Y}, \tau \times \sigma, A)$ is binary soft $P_{0}$ space.

Proof. Let $(\tilde{X}, \tilde{Y}, \tau \times \sigma, A)$ is binary soft $P_{0}$ space with respect to first and the second coordinates. Let $\left(x_{1}, y_{1}\right),\left(x_{2}, y_{2}\right) \tilde{E} X \times Y$ with $x_{1}>x_{2}, y_{1}>$ $y_{2}$.Take $\alpha \tilde{\varepsilon} Y$ and $\beta \tilde{\varepsilon} X$.Then $\left(x_{1}, \alpha\right),\left(x_{2}, \alpha\right) \tilde{\mathcal{E}} X \times Y$.Since $(\tilde{X}, \tilde{Y}, \tau \times \sigma, A)$ is a binary soft $P_{0}$ space with respect to the first coordinate, by using definition, there exists $((F, A),(G, A)) \tilde{\varepsilon} \tau \times \sigma$ with $x_{1} \tilde{\varepsilon}(F, A), x_{2} \widetilde{\notin}(F, A), \alpha \tilde{\varepsilon}(G, A)$. Since $\left(\beta, y_{1}\right),\left(\beta, y_{2}\right) \tilde{\varepsilon} X \times Y$, by using the arguments and using definition there $\quad$ exists $\quad((H, A),(K, A)) \tilde{\mathcal{E}} \tau \times \sigma \quad$ with $y_{1} \tilde{\varepsilon}(K, A), y_{1} \widetilde{\notin}(K, A), \widetilde{\beta \varepsilon}(H, A)$.Therefore, $\left(x_{1}, y_{1}\right) \tilde{\varepsilon}((F, A),(K, A))$ and $\left(x_{2}, y_{2}\right) \tilde{\mathcal{E}}\left(\left(F^{C}, A\right),\left(K^{C}, A\right)\right)$. Hence $(\tilde{X}, \tilde{Y}, \tau \times \sigma, A)$ is called a binary soft b$T_{0}$

Theorem 3. A binary soft topological space $(\tilde{X}, \tau, A)$ and $(\tilde{Y}, \sigma, A)$ are soft $P_{1}$ spaces if and only if the binary soft topological space $(\tilde{X}, \tilde{Y}, \tau \times \sigma, A)$ is soft binary $P_{1}$.

Proof. Suppose $(\tilde{X}, \tau, A)$ and $(\tilde{X}, \sigma, A)$ are soft $P_{1}$ spaces. Let $\left(x_{1}, y_{1}\right),\left(x_{2}, y_{2}\right) \tilde{\varepsilon} X \times Y$ with $x_{1}>x_{2}, y_{1}>y_{2}$. since $(\tilde{X}, \tau, A)$ is soft $P_{1}$ space, there exists soft p-open sets such that $(F, A),(G, A) \tilde{\mathcal{E}} \tau, x_{1} \varepsilon(F, A)$ and $x_{2} \varepsilon(G, A)$ such that $x_{1} \widetilde{\notin}(G, A)$ and $x_{2} \widetilde{\notin}(F, A)$. Also, since $(\tilde{Y}, \sigma, A)$ is soft $P_{1}$ space, there exists soft p-open sets such that $(H, A),(I, A) \tilde{\varepsilon} \sigma, y_{1} \varepsilon(H, A)$ and $y_{2} \varepsilon(I, A)$ such that $y_{1} \widetilde{\notin}(I, A)$ and $y_{2} \widetilde{\notin}(H, A)$.thus $\left(x_{1}, y_{1}\right) \mathcal{E}((F, A),(H, A))$ and $\left(x_{2}, y_{2}\right) \mathcal{E}((G, A),(I, A))$ with $\left(x_{1}, y_{1}\right) \mathcal{E}\left(\left(G^{C}, A\right),\left(I^{C}, A\right)\right)$ and $\left(x_{1}, y_{1}\right) \mathcal{E}\left(\left(F^{C}, A\right),\left(H^{C}, A\right)\right)$.This implies that $(\tilde{X}, \tilde{Y}, \tau \times \sigma, A)$ is soft binary $P_{1}$. Conversely assume that $(\tilde{X}, \tilde{Y}, \tau \times \sigma, A)$ is soft binary $P_{1}$. Let $x_{1}, x_{2} \varepsilon X$ and $y_{1}, y_{2} \varepsilon Y$ such that $x_{1}>x_{2}, y_{1}>y_{2}$. Therefore $\left(x_{1}, y_{1}\right),\left(x_{2}, y_{2}\right) \tilde{\varepsilon} X \times Y$. Since $(\tilde{X}, \tilde{Y}, \tau \times \sigma, A)$ is soft binary $P_{1}$, there exists $(F, A),(G, A)$ and $(H, A),(I, A) \mathcal{E}(\tau \times$ $\sigma),\left(x_{1}, y_{1}\right) \tilde{\mathcal{E}}((F, A),(G, A))$ and $\left(x_{1}, y_{1}\right) \tilde{\mathcal{E}}((H, A),(I, A))$ such that $\left(x_{1}, y_{1}\right) \mathcal{E}\left(H^{C}, \bar{A}\right),\left(I^{C}, A\right) \quad$ and $\quad\left(x_{2}, y_{2}\right) \tilde{\mathcal{E}}\left(\left(F^{C}, A\right),\left(G^{C}, A\right)\right)$. Therefore, $x_{1} \varepsilon(F, A), x_{2} \varepsilon(H, A)$ and $x_{1} \varepsilon\left(H^{C}, A\right)$ and $x_{2} \varepsilon\left(F^{C}, A\right)$ and , $y_{1} \varepsilon\left(G^{C}, A\right)$ and $y_{1} \varepsilon(I, A)$ and , $y_{1} \varepsilon\left(I^{C}, A\right)$ and , $y_{2} \varepsilon\left(G^{C}, A\right)$. Since $(F, A),(G, A) \tilde{\mathcal{E}} \tau \times \sigma$, We have $(F, A),(H, A) \mathcal{E} \tau$ and $(G, A),(I, A) \mathcal{E} \sigma$. This proves that $(\tilde{X}, \tau, A)$ and $(\tilde{X}, \sigma, A)$ are soft $P_{1}$ spaces.

Theorem 4. A binary soft topological space $(\tilde{X}, \tilde{Y}, \mathcal{M}, A)$ is binary soft $P_{1}$ space if and only if every binary soft point $\wp(X) \times \wp(Y)$ is binary soft p-closed.

Proof. Suppose that $(\tilde{X}, \tilde{Y}, \mathcal{M}, A)$ is binary soft $P_{1}$ space. $\operatorname{Let}(x, y) \tilde{\varepsilon} X \times Y$. Let $(\{x\},\{y\}) \tilde{\varepsilon} \wp(X) \times \wp(Y)$.We shall show that $(\{x\},\{y\})$ is binary soft $\mathrm{p}$ closed.it is sufficient to show that $(X \backslash\{x\}, Y \backslash\{y\})$ is binary soft p-open. Let $(a, b) \varepsilon(X \backslash\{x\}, Y \backslash\{y\})$. This implies that $a \tilde{\varepsilon} X \backslash\{x\}$ and $b \tilde{\varepsilon} Y \backslash\{y\}$. hence $a \neq$ 
$x$ and $b \neq y$.That is, $(a, b)$ and $(x, y)$ are distinct binary soft points of $X \times Y$. Since $(\tilde{X}, \tilde{Y}, \mathcal{M}, A)$ is binary soft $P_{1}$ space, there exists binary soft $\mathrm{p}$ open sets $((F, A),(G, A))$ and $(H, A),(I, A)$ such that $(a, b) \mathcal{E}((F, A),(G, A))$ and $(x, y) \mathcal{E}((H, A),(I, A))$ such that $(a, b) \varepsilon\left(\left(H^{C}, A\right),\left(I^{C}, A\right)\right)$ and $(x, y) \varepsilon\left(\left(F^{C}, A\right),\left(G^{C}, A\right)\right)$. Therefore, $\quad((F, A),(G, A)) \subseteq\left(\{x\}^{C},\{y\}^{c}\right)$. Hence $\left(\{x\}^{c},\{y\}^{c}\right)$ is a soft neighbourhood of $(a, b)$. This implies that $(\{x\},\{y\})$ is binary soft p-closed. Conversely, suppose that $(\{x\},\{y\})$ is binary soft b-closed for every $(x, y) \varepsilon X \times Y$.Suppose $\left(x_{1}, y_{1}\right),\left(x_{2}, y_{2}\right) \tilde{\varepsilon} X \times$ $Y \quad$ with $x_{1}>x_{2}, y_{1}>y_{2}$.Therefore, $\quad\left(x_{2}, y_{2}\right) \mathcal{E}\left(\left\{x_{1}\right\}^{c},\left\{y_{1}\right\}^{c}\right)$ and $\mathcal{E}^{\prime}\left(\left\{x_{1}\right\}^{c},\left\{y_{1}\right\}^{c}\right)$ is binary soft p-open. Also $\left(x_{1}, y_{1}\right) \mathcal{E}\left(\left\{x_{2}\right\}^{c},\left\{y_{2}\right\}^{c}\right)$ and $\mathcal{E}\left(\left\{x_{1}\right\}^{c},\left\{y_{1}\right\}^{c}\right)$ is binary soft p-open set. Also $\left(x_{1}, y_{1}\right) \mathcal{E}\left(\left\{x_{2}\right\}^{c},\left\{y_{2}\right\}^{c}\right)$ and $\mathcal{E}\left(\left\{x_{2}\right\}^{c},\left\{y_{2}\right\}^{c}\right)$ is binary soft p-open set. This shows that $(\tilde{X}, \tilde{Y}, \mathcal{M}, A)$ is binary soft $P_{1}$ space.

Theorem 5. A binary soft topological space $(\tilde{X}, \tau, A)$ and $(\tilde{Y}, \sigma, A)$ are soft $P_{2}$ spaces if and only if the binary soft topological space $(\tilde{X}, \tilde{Y}, \tau \times \sigma, A)$ is soft binary $P_{2}$.

Proof. Suppose $(\tilde{X}, \tau, A)$ and $(\tilde{X}, \sigma, A)$ are soft $P_{2}$ spaces. Let $\left(x_{1}, y_{1}\right),\left(x_{2}, y_{2}\right) \tilde{E} X \times Y$ with $x_{1}>x_{2}, y_{1}>y_{2}$. since $(\tilde{X}, \tau, A)$ is soft $P_{2}$ space, there exists soft p-open sets such that $(F, A),(G, A) \tilde{\mathcal{E}} \tau, x_{1} \mathcal{E}(F, A)$ and $x_{2} \mathcal{E}(G, A)$ such that $x_{1} \widetilde{\notin}(G, A)$ and $x_{2} \widetilde{\notin}(F, A)$. Also, since $(\tilde{Y}, \sigma, A)$ is soft $P_{2}$ space, there exists disjoint soft p-open sets such that $(H, A),(I, A) \tilde{\varepsilon} \sigma, y_{1} \varepsilon(H, A)$ and $y_{2} \varepsilon(I, A)$ such that $y_{1} \widetilde{\notin}(I, A)$ and $y_{2} \widetilde{\notin}(H, A)$.thus $\left(x_{1}, y_{1}\right) \mathcal{E}((F, A),(H, A))$ and $\left(x_{2}, y_{2}\right) \mathcal{E}((G, A),(I, A))$ with $\left(x_{1}, y_{1}\right) \mathcal{E}\left(\left(G^{C}, A\right),\left(I^{C}, A\right)\right) \quad$ and $\quad\left(x_{1}, y_{1}\right) \mathcal{E}\left(\left(F^{C}, A\right),\left(H^{C}, A\right)\right)$. Snce $(F, A)$ and $(G, A)$ are disjoint, $(F, A) \sqcap(H, A)=(\Phi, \Phi)$. . Also since $(H, A) \sqcap(I, A)=(\Phi, \Phi) . \quad \operatorname{Thus}((F, A) \sqcap(H, A),(G, A) \sqcap(I, A))=$ $(\Phi, \Phi)$. This implies that we have this implies that $(\tilde{X}, \tilde{Y}, \tau \times \sigma, A)$ is soft binary $P_{2}$. Conversely assume that $(\tilde{X}, \tilde{Y}, \tau \times \sigma, A)$ is soft binary $P_{2}$. Let $x_{1}, x_{2} \varepsilon X$ and $y_{1}, y_{2} \varepsilon Y$ such that $x_{1}>x_{2}, y_{1}>y_{2}$. Therefore $\left(x_{1}, y_{1}\right),\left(x_{1}, y_{1}\right) \tilde{E} X \times Y$. Since $(\tilde{X}, \tilde{Y}, \tau \times \sigma, A)$ is soft binary $P_{2}$, there exists binary soft p-open sets $(F, A),(G, A)$ and there exists binary soft b-open sets $\quad(H, A),(I, A) \mathcal{E}(\tau \times \sigma),\left(x_{1}, y_{1}\right) \tilde{\mathcal{E}}((F, A),(G, A)) \quad$ and $\left(x_{2}, y_{2}\right) \tilde{\mathcal{E}}((\mathrm{H}, A),(I, A))$ such that $\left(x_{1}, y_{1}\right) \mathcal{E}\left(H^{C}, \bar{A}\right),\left(I^{C}, A\right)$ and $\left(x_{2}, y_{2}\right) \tilde{\mathcal{E}}\left(\left(F^{C}, A\right),\left(G^{C}, A\right)\right)$. Therefore, $x_{1} \varepsilon(F, A), x_{2} \varepsilon(H, A)$ and $x_{1} \mathcal{E}\left(H^{C}, A\right)$ and $x_{2} \varepsilon\left(F^{C}, A\right)$ and,$y_{1} \varepsilon\left(G^{C}, A\right)$ and $y_{2} \varepsilon(I, A)$ and,$y_{1} \varepsilon\left(I^{C}, A\right)$ and ,$y_{2} \mathcal{E}\left(G^{C}, A\right)$. Since $(F, A),(G, A) \tilde{\mathcal{E}} \tau \times \sigma$, We have $(F, A),(H, A) \mathcal{E} \tau$ and $(G, A),(I, A) \mathcal{E} \sigma$. This proves that $(\tilde{X}, \tau, A)$ and $(\tilde{X}, \sigma, A)$ are soft $\mathrm{b}-T_{2}$ spaces.

\section{BINARY SOFT STRUCTURES WITH RESPECT TO SOFT POINTS}

Theorem 6. If the binary soft topological space $(\tilde{X}, \tilde{Y}, \rho \times \sigma, A)$ is a binary soft $\mathrm{P}_{0}$, then $(\tilde{X}, \rho, A)$ and $(\tilde{Y}, \sigma, A)$ are soft $\mathrm{P}_{0}$.

Proof. We suppose $\left(\widetilde{X_{A}}, \widetilde{\widetilde{Y}_{A}}, \rho \times \sigma, A\right)$ is a binary soft $\mathrm{P}_{0}$. Suppose $e_{\mathbb{G}_{1}}, e_{\mathbb{G}_{2}} \tilde{\varepsilon} \widetilde{X}_{A}$ and $e_{\mathbb{H}_{1}}, e_{\mathbb{H}_{2}} \tilde{\varepsilon} \widetilde{Y}_{A}$ with such that $e_{\mathbb{G}_{1}}>e_{\mathbb{G}_{2}}, e_{\mathbb{H}_{1}}>e_{\mathbb{H}_{2}}$. Since $\left(\widetilde{X_{A}}, \widetilde{\widetilde{Y}}_{A}, \rho \times \sigma, A\right)$ is a binary soft $\mathrm{P}_{0}$, accordingly there binary soft p-open set $((F, A),(G, A))$ such

that $\left(e_{\mathbb{G}_{1}}, e_{\mathbb{H}_{1}}\right) \tilde{\mathcal{E}}((F, A),(G, A)) ;\left(e_{\mathbb{G}_{2}}, e_{\mathbb{H}_{2}}\right) \tilde{\mathcal{E}}\left(F^{C}, A\right),\left(G^{C}, A\right)$

$\left(e_{\mathbb{G}_{1}}, e_{\mathbb{H}_{1}}\right) \tilde{\varepsilon}\left(\left(F^{C}, A\right),\left(G^{C}, A\right)\right) ;\left(e_{\mathbb{G}_{2}}, e_{\mathbb{H}_{2}}\right) \tilde{\varepsilon}((F, A),(G, A))$. This implies that eithere $_{\mathbb{G}_{1}} \tilde{\varepsilon}(F, A) ; e_{\mathbb{G}_{2}} \tilde{\varepsilon}\left(F^{C}, A\right) ; e_{\mathbb{H}_{1}} \tilde{\varepsilon}(G, A) ; e_{\mathbb{H}_{2}} \tilde{\varepsilon}\left(G^{C}, A\right)$;or

$e_{\mathbb{G}_{1}} \tilde{\varepsilon}\left(F^{C}, A\right) ; e_{\mathbb{H}_{1}} \tilde{\varepsilon}\left(G^{C}, A\right) ; e_{\mathbb{H}_{2}} \tilde{\varepsilon}(G, A)$. This implies either $e_{\mathbb{G}_{1}} \tilde{\varepsilon}(F, A) ; e_{\mathbb{G}_{2}} \tilde{\varepsilon}\left(F^{C}, A\right)$ or $\quad e_{\mathbb{G}_{1}} \tilde{\varepsilon}\left(F^{C}, A\right) ; x_{1} \tilde{\varepsilon}(F, A)$ and $\quad$ either $e_{\mathbb{H}_{1}} \tilde{\varepsilon}(G, A) ; e_{\mathbb{H}_{2}} \tilde{\varepsilon}\left(G^{C}, A\right) \operatorname{ore}_{\mathbb{H}_{1}} \tilde{\varepsilon}\left(G^{C}, A\right) ; e_{\mathbb{H}_{2}} \tilde{\varepsilon}(G, A)$. Since $((F, A),(G, A)) \tilde{\mathcal{E}} \rho \times \sigma$, We have p-open $(F, A) \tilde{\varepsilon} \rho$ and p-open $(F, A) \widetilde{\varepsilon} \sigma$. this proves that $(\tilde{X}, \rho, A)$ and $(\tilde{Y}, \sigma, A)$ are soft $\mathrm{P}_{0}$.

Theorem 7. A binary soft topological space $(\tilde{X}, \tilde{Y}, \tau \times \sigma, A)$ is binary soft $\mathrm{P}_{0}$ space with respect to first and the second coordinates, then $(\tilde{X}, \tilde{Y}, \tau \times$ $\sigma, A$ )is binary soft $\mathrm{P}_{0}$ space.

Proof. Let $(\tilde{X}, \tilde{Y}, \tau \times \sigma, A)$ is binary soft $\mathrm{P}_{0}$ space with respect to first and the second coordinates. Let $\left(e_{\mathbb{G}_{1}}, e_{\mathbb{H}_{1}}\right),\left(e_{\mathbb{G}_{2}}, e_{\mathbb{H}_{2}}\right) \tilde{\varepsilon} X \times Y$ with $e_{\mathbb{G}_{1}}>$ $e_{\mathbb{G}_{2}}, e_{\mathbb{H}_{1}}>e_{\mathbb{H}_{2}}$. Take $\alpha \tilde{\varepsilon} Y$ and $\beta \tilde{\varepsilon} X$.Then $\left(e_{\mathbb{G}_{1}}, \alpha\right),\left(e_{\mathbb{G}_{2}}, \alpha\right) \tilde{\varepsilon} X \times Y$. Since $(\tilde{X}, \tilde{Y}, \tau \times \sigma, A)$ is a binary soft $\mathrm{P}_{0}$ space with respect to the first coordinate, by using definition, there exists $((F, A),(G, A)) \tilde{\mathcal{E}} \tau \times \sigma$ with $e_{\mathbb{G}_{1}} \tilde{\varepsilon}(F, A)$, $e_{\mathbb{G}_{2}} \widetilde{\notin}(F, A), \quad \alpha \tilde{\varepsilon}(G, A)$. Since $\left(\beta, e_{\mathbb{H}_{1}}\right),\left(\beta, e_{\mathbb{H}_{2}}\right) \tilde{\varepsilon} X \times Y$, by using the arguments and using definition there exists $((H, A),(K, A)) \tilde{\varepsilon} \tau \times \sigma$ with $e_{\mathbb{H}_{1}} \tilde{\varepsilon}(K, A), e_{\mathbb{H}_{1}} \widetilde{\notin}(K, A), \widetilde{\beta \varepsilon}(H, A)$ where $(H, A)$ is p-open in $\tau$ and $(K, A)$ is p-open in $\sigma$ Therefore, $\left(e_{\mathbb{G}_{1}}, e_{\mathbb{H}_{1}}\right) \tilde{\varepsilon}((F, A),(K, A))$ and $\left(e_{\mathbb{G}_{2}}, e_{\mathbb{H}_{2}}\right) \tilde{\mathcal{E}}\left(\left(F^{C}, A\right),\left(K^{C}, A\right)\right)$. Hence $(\tilde{X}, \tilde{Y}, \tau \times \sigma, A)$ is called a binary soft $\mathrm{P}_{0}$

Theorem 8. A binary soft topological space $(\tilde{X}, \tau, A)$ and $(\tilde{Y}, \sigma, A)$ are soft $\mathrm{P}_{1}$ spaces if and only if the binary soft topological space $(\tilde{X}, \tilde{Y}, \tau \times \sigma, A)$ is soft binary $\mathrm{P}_{1}$.

Proof. Suppose $(\tilde{X}, \tau, A)$ and $(\tilde{X}, \sigma, A)$ are soft $\mathrm{P}_{1}$ spaces. Let $\left(e_{\mathbb{G}_{1}}, e_{\mathbb{H}_{1}}\right),\left(e_{\mathbb{G}_{2}}, e_{\mathbb{H}_{2}}\right) \tilde{\varepsilon} X \times Y$ with $e_{\mathbb{G}_{1}}>e_{\mathbb{G}_{2}}, e_{\mathbb{H}_{1}}>e_{\mathbb{H}_{2}}$. since $(\tilde{X}, \tau, A)$ is soft $\mathrm{P}_{1}$ space, there exists soft p-open sets such that $(F, A),(G, A) \tilde{\varepsilon} \tau$, $e_{\mathbb{G}_{1}} \mathcal{E}(F, A)$ and $e_{\mathbb{G}_{2}} \mathcal{E}(G, A)$ such that $e_{\mathbb{G}_{1}} \widetilde{\notin}(G, A)$ and $e_{\mathbb{G}_{2}} \widetilde{\notin}(F, A)$. Also, since $(\tilde{Y}, \sigma, A)$ is soft $\mathrm{P}_{1}$ space, there exists soft p-open sets such that $(H, A),(I, A) \tilde{\varepsilon} \sigma, e_{\mathbb{H}_{1}} \mathcal{\varepsilon}(H, A)$ and $e_{\mathbb{H}_{2}} \mathcal{\varepsilon}(I, A)$ such that $e_{\mathbb{H}_{1}} \widetilde{\notin}(I, A)$ and $e_{\mathbb{H}_{2}} \widetilde{\notin}(H, A)$.thus $\left(e_{\mathbb{G}_{1}}, e_{\mathbb{H}_{1}}\right) \mathcal{E}((F, A),(H, A))$ and $\left(e_{\mathbb{G}_{2}}, e_{\mathbb{H}_{2}}\right) \mathcal{E}((G, A),(I, A))$ with $\left(e_{\mathbb{G}_{1}}, e_{\mathbb{H}_{1}}\right) \mathcal{E}\left(\left(G^{C}, A\right),\left(I^{C}, A\right)\right)$ and $\left(e_{\mathbb{G}_{1}}, e_{\mathbb{H}_{1}}\right) \mathcal{E}\left(\left(F^{C}, A\right),\left(H^{C}, A\right)\right)$.This implies that $(\tilde{X}, \tilde{Y}, \tau \times \sigma, A)$ is soft binary $\mathrm{P}_{1}$. Conversely assume that $(\tilde{X}, \tilde{Y}, \tau \times \sigma, A)$ is soft binary $\mathrm{P}_{1}$. Let $e_{\mathbb{G}_{1}}, e_{\mathbb{G}_{2}} \varepsilon X$ and $e_{\mathbb{H}_{1}}, e_{\mathbb{H}_{2}} \varepsilon Y$ such that $e_{\mathbb{G}_{1}}>e_{\mathbb{G}_{2}}, e_{\mathbb{H}_{1}}>e_{\mathbb{H}_{2}}$. Therefore $\left(e_{\mathbb{G}_{1}}, e_{\mathbb{H}_{1}}\right),\left(e_{\mathbb{G}_{2}}, e_{\mathbb{H}_{2}}\right) \tilde{\varepsilon} X \times Y$. Since $(\tilde{X}, \tilde{Y}, \tau \times \sigma, A)$ is soft binary $\mathrm{P}_{1}$, there exists p-open sets $(F, A),(G, A)$ and p-open sets $(H, A),(I, A) \mathcal{E}(\tau \times \sigma),\left(e_{\mathbb{G}_{1}}, e_{\mathbb{H}_{1}}\right) \tilde{\varepsilon}((F, A),(G, A)) \quad$ and $\left(e_{\mathbb{G}_{2}}, e_{\mathbb{H}_{2}}\right) \tilde{\varepsilon}((H, A),(I, A))$ such that $\left(e_{\mathbb{G}_{1}}, e_{\mathbb{H}_{1}}\right) \mathcal{E}\left(H^{C}, \bar{A}\right),\left(I^{C}, A\right)$ and $\left(e_{\mathbb{G}_{2}}, e_{\mathbb{H}_{2}}\right) \tilde{\varepsilon}\left(\left(F^{C}, A\right),\left(G^{C}, A\right)\right)$. Therefore, $e_{\mathbb{G}_{1}} \varepsilon(F, A), e_{\mathbb{G}_{2}} \varepsilon(H, A) \quad$ and $e_{\mathbb{G}_{1}} \mathcal{E}\left(H^{C}, A\right)$ and $e_{\mathbb{G}_{2}} \mathcal{E}\left(F^{C}, A\right)$ and,$y_{1} \mathcal{\varepsilon}\left(G^{C}, A\right)$ and $e_{\mathbb{H}_{1}} \mathcal{E}(I, A)$ and , $e_{\mathbb{H}_{1}} \varepsilon\left(I^{C}, A\right) \quad$ and $\quad, e_{\mathbb{H}_{2}} \varepsilon\left(G^{C}, A\right)$. Since $(F, A),(G, A) \tilde{\mathcal{E}} \tau \times \sigma$, We have $(F, A),(H, A) \mathcal{E} \tau$ and $(G, A),(I, A) \mathcal{E} \sigma$. This proves that $(\tilde{X}, \tau, A)$ and $(\tilde{X}, \sigma, A)$ are soft $P_{1}$ spaces.

Theorem 9. A binary soft topological space $(\tilde{X}, \tilde{Y}, \mathcal{M}, A)$ is binary soft $P_{1}$ space if and only if every binary soft point $\wp(X) \times \wp(Y)$ is binary soft p-closed.

Proof. Suppose that $(\tilde{X}, \tilde{Y}, \mathcal{M}, A)$ is binary soft $P_{1}$ space. Let $(x, y) \tilde{\mathcal{E}} X \times Y$. Let $\left(\{x\},\left\{e_{\mathbb{H}}\right\}\right) \tilde{\mathcal{E}} \wp(X) \times \wp(Y)$.We shall show that $\left(\{x\},\left\{e_{\mathbb{H}}\right\}\right)$ is binary soft p-closed.it is sufficient to show that $\left(X \backslash\left\{e_{\mathbb{G}}\right\}, Y \backslash\left\{e_{\mathbb{H}}\right\}\right)$ is binary soft p-open. Let $(a, b) \mathcal{E}\left(X \backslash\left\{e_{\mathbb{G}}\right\}, Y \backslash\left\{e_{\mathbb{H}}\right\}\right)$. This implies that $a \tilde{\varepsilon} X \backslash\left\{e_{\mathbb{G}}\right\}$ and $b \tilde{\varepsilon} Y \backslash\left\{e_{\mathbb{H}}\right\}$. hence $a \neq e_{\mathbb{G}}$ and $b \neq e_{\mathbb{H}}$. That is, $(a, b)$ and $\left(e_{\mathbb{G}}, e_{\mathbb{H}}\right)$ are distinct binary soft points of $X \times Y$. Since $(\tilde{X}, \tilde{Y}, \mathcal{M}, A)$ is binary soft $P_{1}$ space, there exists binary soft p-open sets $((F, A),(G, A))$ and $(H, A),(I, A)$ such that $(a, b) \varepsilon((F, A),(G, A)) \quad$ and $(x, y) \varepsilon((H, A),(I, A))$ such that $(a, b) \varepsilon\left(\left(H^{C}, A\right),\left(I^{C}, A\right)\right) \quad$ and $\quad\left(e_{\mathbb{G}}, e_{\mathbb{H}}\right) \varepsilon\left(\left(F^{C}, A\right),\left(G^{C}, A\right)\right)$. Therefore, $((F, A),(G, A)) \subseteq\left(\left\{e_{\mathbb{G}}\right\}^{c},\left\{e_{\mathbb{H}}\right\}^{c}\right)$. Hence $\left(\left\{e_{\mathbb{G}}\right\}^{c},\left\{e_{\mathbb{H}}\right\}^{c}\right)$ is a soft neighbourhood of $(a, b)$.This implies that $\left(\left\{e_{\mathbb{G}}\right\},\left\{e_{\mathbb{H}}\right\}\right)$ is binary soft pclosed. Conversely, suppose that $\left(\left\{e_{\mathbb{G}}\right\},\left\{e_{\mathbb{H}}\right\}\right)$ is binary soft p-closed for every $\left(e_{\mathbb{G}}, e_{\mathbb{H}}\right) \varepsilon X \times Y$.Suppose $\left(e_{\mathbb{G}_{1}}, e_{\mathbb{H}_{1}}\right),\left(e_{\mathbb{G}_{2}}, e_{\mathbb{H}_{2}}\right) \tilde{\varepsilon} X \times Y$ with $e_{\mathbb{G}_{1}}>$ $e_{\mathbb{G}_{2}}, e_{\mathbb{H}_{1}}>e_{\mathbb{H}_{2}}$. Therefore, $\quad\left(e_{\mathbb{G}_{2}}, e_{\mathbb{H}_{2}}\right) \mathcal{E}\left(\left\{e_{\mathbb{G}_{1}}\right\}^{c},\left\{e_{\mathbb{H}_{1}}\right\}^{c}\right)$ and $\mathcal{E}^{\prime}\left(\left\{e_{\mathbb{G}_{1}}\right\}^{c},\left\{e_{\mathbb{H}_{1}}\right\}^{c}\right)$ is binary soft p-open. Also $\left(e_{\mathbb{G}_{1}}, e_{\mathbb{H}_{1}}\right) \mathcal{E}\left(\left\{e_{\mathbb{G}_{2}}\right\}^{c},\left\{e_{\mathbb{H}_{2}}\right\}^{c}\right)$ and $\mathcal{E}\left(\left\{e_{\mathbb{G}_{1}}\right\}^{c},\left\{e_{\mathbb{H}_{1}}\right\}^{c}\right)$ is binary soft p-open set. Also $\left(e_{\mathbb{G}_{1}}, e_{\mathbb{H}_{1}}\right) \mathcal{E}\left(\left\{e_{\mathbb{G}_{2}}\right\}^{c},\left\{e_{\mathbb{H}_{2}}\right\}^{c}\right)$ and $\mathcal{E}\left(\left\{e_{\mathbb{G}_{2}}\right\}^{c},\left\{e_{\mathbb{H}_{2}}\right\}^{c}\right)$ is binary soft p-open set. This shows that $(\tilde{X}, \tilde{Y}, \mathcal{M}, A)$ is binary soft $P_{1}$ space.

Theorem 10. A binary soft topological space $(\tilde{X}, \tau, A)$ and $(\tilde{Y}, \sigma, A)$ are soft $P_{2}$ spaces if and only if the binary soft topological space $(\tilde{X}, \tilde{Y}, \tau \times \sigma, A)$ is soft binary $P_{2}$.

Proof. Suppose $(\tilde{X}, \tau, A)$ and $(\tilde{X}, \sigma, A)$ are soft $P_{2}$ spaces. Let $\left(e_{\mathbb{G}_{1}}, e_{\mathbb{H}_{1}}\right),\left(e_{\mathbb{G}_{2}}, e_{\mathbb{H}_{2}}\right) \tilde{\varepsilon} X \times Y$ with $e_{\mathbb{G}_{1}}>e_{\mathbb{G}_{2}}, e_{\mathbb{H}_{1}}>e_{\mathbb{H}_{2}}$. since $(\tilde{X}, \tau, A)$ is soft $P_{2}$ space, there exists soft p-open sets such that $(F, A),(G, A) \tilde{\mathcal{E}} \tau$, $e_{\mathbb{G}_{1}} \varepsilon(F, A)$ and $e_{\mathbb{G}_{2}} \varepsilon(G, A)$ such that $e_{\mathbb{G}_{1}} \widetilde{\notin}(G, A)$ and $e_{\mathbb{G}_{2}} \widetilde{\notin}(F, A)$. Also, since $(\tilde{Y}, \sigma, A)$ is soft $P_{2}$ space, there exists distoint soft p-open sets such that $(H, A),(I, A) \tilde{\varepsilon} \sigma, e_{\mathbb{H}_{1}} \mathcal{E}(H, A)$ and $e_{\mathbb{H}_{2}} \mathcal{E}(I, A)$ such that $e_{\mathbb{H}_{1}} \widetilde{\notin}(I, A)$ and $e_{\mathbb{H}_{2}} \widetilde{\notin}(H, A)$.thus $\left(e_{\mathbb{G}_{1}}, e_{\mathbb{H}_{1}}\right) \mathcal{\varepsilon}((F, A),(H, A))$ and $\left(e_{\mathbb{G}_{2}}, e_{\mathbb{H}_{2}}\right) \mathcal{E}((G, A),(I, A))$ with $\quad\left(e_{\mathbb{G}_{1}}, e_{\mathbb{H}_{1}}\right) \mathcal{E}\left(\left(G^{C}, A\right),\left(I^{C}, A\right)\right) \quad$ and $\left(e_{\mathbb{G}_{1}}, e_{\mathbb{H}_{1}}\right) \mathcal{E}\left(\left(F^{C}, A\right),\left(H^{C}, A\right)\right)$.Snce $(F, A)$ and $(G, A)$ are disjoint, $(F, A) \Pi$ $(H, A)=(\Phi, \Phi)$. Also $\operatorname{since}(H, A) \sqcap(I, A)=(\Phi, \Phi) . \quad \operatorname{Thus}((F, A) \sqcap$ $(H, A),(G, A) \sqcap(I, A))=(\Phi, \Phi)$. This implies that we have this implies that $(\tilde{X}, \tilde{Y}, \tau \times \sigma, A)$ is soft binary $P_{2}$. Conversely assume that $(\tilde{X}, \tilde{Y}, \tau \times$ $\sigma, A)$ is soft binary $P_{2}$. Let $e_{\mathbb{G}_{1}}, e_{\mathbb{G}_{2}} \varepsilon X$ and $e_{\mathbb{H}_{1}}, e_{\mathbb{H}_{2}} \varepsilon Y$ such that $e_{\mathbb{G}_{1}}>$ $e_{\mathbb{G}_{2}}, e_{\mathbb{H}_{1}}>e_{\mathbb{H}_{2}}$ Therefore $\left(e_{\mathbb{G}_{1}}, e_{\mathbb{H}_{1}}\right),\left(e_{\mathbb{G}_{2}}, e_{\mathbb{H}_{2}}\right) \tilde{\varepsilon} X \times Y$. Since $(\tilde{X}, \tilde{Y}, \tau \times$ $\sigma, A)$ is soft binary $P_{2}$ there exists binary soft p-open sets $(F, A),(G, \mathrm{~A})$ and there exists binary soft p-open sets $(\mathrm{H}, \mathrm{A}),(\mathrm{I}, \mathrm{A}) \mathcal{E}(\tau \times$ $\sigma),\left(e_{\mathbb{G}_{1}}, e_{\mathbb{H}_{1}}\right) \tilde{\mathcal{E}}((\mathrm{F}, \mathrm{A}),(\mathrm{G}, \mathrm{A}))$ and $\left(e_{\mathbb{G}_{2}}, e_{\mathbb{H}_{2}}\right) \tilde{\varepsilon}((\mathrm{H}, \mathrm{A}),(\mathrm{I}, \mathrm{A}))$ such that $\left(e_{\mathbb{G}_{1}}, e_{\mathbb{H}_{1}}\right) \varepsilon\left(\mathrm{H}^{\mathrm{C}}, \overline{\mathrm{A}}\right),\left(\mathrm{I}^{\mathrm{C}}, \mathrm{A}\right) \quad$ and $\quad\left(e_{\mathbb{G}_{2}}, e_{\mathbb{H}_{2}}\right) \tilde{\varepsilon}\left(\left(\mathrm{F}^{\mathrm{C}}, \mathrm{A}\right),\left(\mathrm{G}^{\mathrm{C}}, \mathrm{A}\right)\right)$ Therefore, $e_{\mathbb{G}_{1}} \varepsilon(\mathrm{F}, \mathrm{A}), e_{\mathbb{G}_{2}} \varepsilon(\mathrm{H}, \mathrm{A})$ and $e_{\mathbb{G}_{1}} \varepsilon\left(\mathrm{H}^{\mathrm{C}}, \mathrm{A}\right)$ and $e_{\mathbb{G}_{2}} \varepsilon\left(\mathrm{F}^{\mathrm{C}}, \mathrm{A}\right)$ and , $e_{\mathbb{H}_{1}} \varepsilon\left(\mathrm{G}^{\mathrm{C}}, \mathrm{A}\right)$ and $e_{\mathbb{H}_{2}} \varepsilon(\mathrm{I}, \mathrm{A})$ and,$e_{\mathbb{H}_{1}} \varepsilon\left(\mathrm{I}^{\mathrm{C}}, \mathrm{A}\right)$ and,$e_{\mathbb{H}_{2}} \varepsilon\left(\mathrm{G}^{\mathrm{C}}, \mathrm{A}\right)$. Since $(\mathrm{F}, \mathrm{A}),(\mathrm{G}, \mathrm{A}) \tilde{\mathcal{E}} \tau \times \sigma$, We have $(\mathrm{F}, \mathrm{A}),(\mathrm{H}, \mathrm{A}) \mathcal{E} \tau$ and $(\mathrm{G}, \mathrm{A}),(\mathrm{I}, \mathrm{A}) \mathcal{E} \sigma$. This proves that $(\widetilde{\mathrm{X}}, \tau, \mathrm{A})$ and $(\widetilde{\mathrm{X}}, \sigma, \mathrm{A})$ are soft $P_{2}$ spaces. 


\section{CONCLUSION}

The soft Separation Axioms namely, $\mathrm{T}_{0}, \mathrm{~T}_{1}-\mathrm{T}_{2}$ are extended to binary soft $\mathrm{P}-\mathrm{T}_{0}, \mathrm{P}-\mathrm{T}_{1}$ and $\mathrm{P}-\mathrm{T}_{2}$ structures with respect to first and second coordinates.

\section{REFERENCES}

[1] Molodtsov, D. 1999. Soft Set Theory First Results. Computers and Mathematics with Applications, 37, 19 - 31.

[2] Molodtsov, D., Leonov, V.Y., Kovkov, D.V. 2006. Soft sets technique and its Application. Nechetkie Sistemy Myagkie Vychisleniya, 1 (1), 8-39.

[3] Acikg ö z, A., Tas, N. 2016. Binary Soft Set Theory. European Journal of Pure and Applied Mathematics, 9 (4), 452- 463.

[4] Benchalli, S.S., Patil, P.G., Dodamani, A.S., Pradeepkumar, J. 2017. On Binary Soft Separation Axioms in Binary Soft Topological Spaces. Global journal and Applied Mathematics, 13 (9), 5393-5412.

[5] Benchalli, S.S., Patil, G., Dodamani, A.S., Kumar, J.P. 2017. On Binary Soft Topological Spaces. International Journal of Applied Mathematics, 30 (6), 437-453.
[6] Kalaichelvi, A., Malini, P.H. 2011. Application of Fuzzy Soft Sets to Investment Decision Making Problem. International Journal of Mathematical Sciences and Applications, 1 (3), 1583-1586.

[7] Özgür, N.Y., Taş, N. 2015. A Note on Application of Fuzzy Soft Sets to Investment Decision Making Problem. Journal of New Theory, 7, 1-10.

[8] Taş, N., Özgür, N.Y., Demir, P. 2017. An Application of Soft Set and Fuzzy Soft Set Theories to Stock Management. Süleyman Demirel University Journal of Natural and Applied Sciences, 21 (2), 791-196.

[9] Alcantud, J.C.R., Rambaud, S.C., Torrecillas, M.J.M. 2017. Valuation Fuzzy Soft Sets: A Flexible Fuzzy Soft Set Based Decision-Making Procedure for the Valuation of Assets. Symmetry, 9 (11), 253; doi:10.3390/sym 9110253

[10] Çağman, N., Enginoğlu, S. 2010. Soft Matrix Theory and Its Decision Making. Computers and Mathematics with Applications, 59, 3308-3314.

[11] Shabir, M., Naz, M. 2011. On Some New Operations in Soft Set Theory. Computers and Mathematics with Applications, 57, 1786-1799. 